Original Research Paper

\title{
Research Impact, the 'New Academic Capital': An Environmental Scan of Research Impact Indicators and Resources for the Humanities and Social Sciences across 32 Countries
}

\author{
Samantha Shewchuk and Amanda Cooper \\ Faculty of Education, Queen's University, Canada
}

Article history

Received: 18-09-2017

Revised: $27-12-2017$

Accepted: 26-01-2018

Corresponding Author: Samantha Shewchuk Faculty of Education, Queen's University, Canada Email: 0sjs3@queensu.ca

\begin{abstract}
Research impact agendas are gaining momentum globally and changing research policies from funding agencies and universities. This article reports on an environmental scan of research impact indicators and resources for Humanities and Social Sciences (HSS) from 32 countries. Changing policies from national research funders include new expectations for researchers to mobilize their research to non-academic audiences that could benefit from its use and demonstrate the tangible impacts of their work. Many have argued that research impact agendas disadvantage HSS as compared to Science, Technology, Engineering and Mathematics (STEM) fields. As such, the purpose of this environmental scan was threefold: (a) to examine how funding agencies are defining and conceptualizing research impact and $\mathrm{KMb}$ in different countries, (b) to gather and analyze research impact indicators used to assess HSS and (c) to identify practical resources that might support HSS researchers with research mobilization and impact. The scan yielded 721 research impact resources relevant to HSS; included analysis of 1,105 indicators; and identified 87 resources for researchers (including tools, networks, projects and open access repositories). Supplementary files for this article include a taxonomy of research impact indicators as well as a guidebook of research impact resources for researchers.
\end{abstract}

Keywords: Environmental Scan, Research Impact, Knowledge Mobilization

\section{Introduction}

Global interest in the field of Knowledge Mobilization (KMb) and Knowledge Translation (KT) (Nutley et al., 2007) has grown alongside the rise of the research impact agenda. $\mathrm{KMb}$ is about how research finds its way (or fails to finds its way) into the hands of those in communities who could benefit from its use. There is a widely acknowledged gap between research, policy, and practice across sectors (Davies, 2003). Research has often failed to have the impact it might due to a lack of capacity to translate and mobilize the work to end users in non-academic settings, including policy makers, practitioners, and community members. The rationale for prioritizing $\mathrm{KMb}$ is persuasive. Historical applications of evidence in countless areas of social policy have seen improved outcomes and benefits for citizens in society, such as hand-washing in health, use of seat belts in transportation, anti-smoking legislation in certain jurisdictions such as Canada, among many others (Cooper et al., 2009). Therefore, it seems obvious people would agree that applications of research, when they have the potential to benefit society, should be prioritized. Chandler (2014) highlighted that:

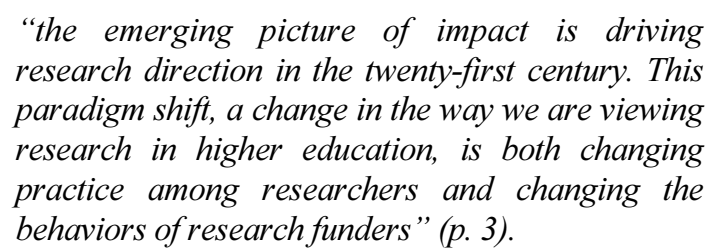
research direction in the twenty-first century. This paradigm shift, a change in the way we are viewing research in higher education, is both changing practice among researchers and changing the behaviors of research funders" (p. 3).

Watermeyer referred to research impact as "new academic capital" (p. 361). Many factors drive the research impact agenda, which Wilsdon et al. (2015) referred to as the "Metric Tide" in higher education: 
"There are powerful currents whipping up the metric tide. These include growing pressures for audit and evaluation of public spending on higher education and research; demands by policymakers for more strategic intelligence on research quality and impact; the need for institutions to manage and develop their strategies for research; competition within and between institutions for prestige, students, staff and resources; and increases in the availability of real-time "big data" on research uptake and the capacity of tools for analyzing them" (p. 5).

Although the focus on impact, research mobilization, and uptake has increased considerably, it is still a contentious area that remains underdeveloped. Also, while empirical exploration of research impact is growing, the effects of large-scale performance-based research funding systems such as the Research Excellence Framework (REF) in the United Kingdom are still relatively unknown. It is unlikely that research impact and metrics will go away, so it is important that we work collaboratively across disciplines to explore the metrics currently in use (which are widely acknowledged to be underdeveloped) and suggest different approaches to measuring and tracing the value of HSS research. Watermeyer (2014) suggested the first step to better understanding the impact phenomenon is to create a cartography of the landscape. Therefore, the contribution this environmental scan makes is to provide descriptive mapping of the research impact landscape across 32 countries.

\section{Research Questions}

The research questions that guided the scan and analysis of research impact resources were the following:
- How are social science research funding agencies defining and conceptualizing research impact and $\mathrm{KMb}$ in different countries?

- What frequency of research impact resources exists for the humanities and social science disciplines?

- What are the attributes (date produced, type of resource, agency of origin, field) of the research impact resources?

- What research impact indicators exist for HSS in relation to six areas: Scholarship, capacity building in teaching and learning, economy, society and culture, practice and policy?

\section{Conceptual Framework}

We used four dimensions to explore the research impact landscape: (a) country of origin, (b) social science funders' mission statements and conceptualization of research impact and $\mathrm{KMb}$ (or related terms), (c) attributes, and (d) indicators (Fig. 1).

The first dimension of the framework explicitly explores research impact across 32 countries to identify global trends in relation to research impact. The second area of the framework situates the broader infrastructure of each of the 32 countries by exploring the missions of social science funding agencies as well as how funders are defining and conceptualizing research impact and $\mathrm{KMb}$ (or related terms). Funders shape national research agendas through explicit requirements for researchers in grant levers (Tetroe et al., 2008); hence, exploring how social science funders in each country conceptualize research impact is an important factor in understanding the landscape of each country and, more globally, how impact agendas are operationalized internationally.

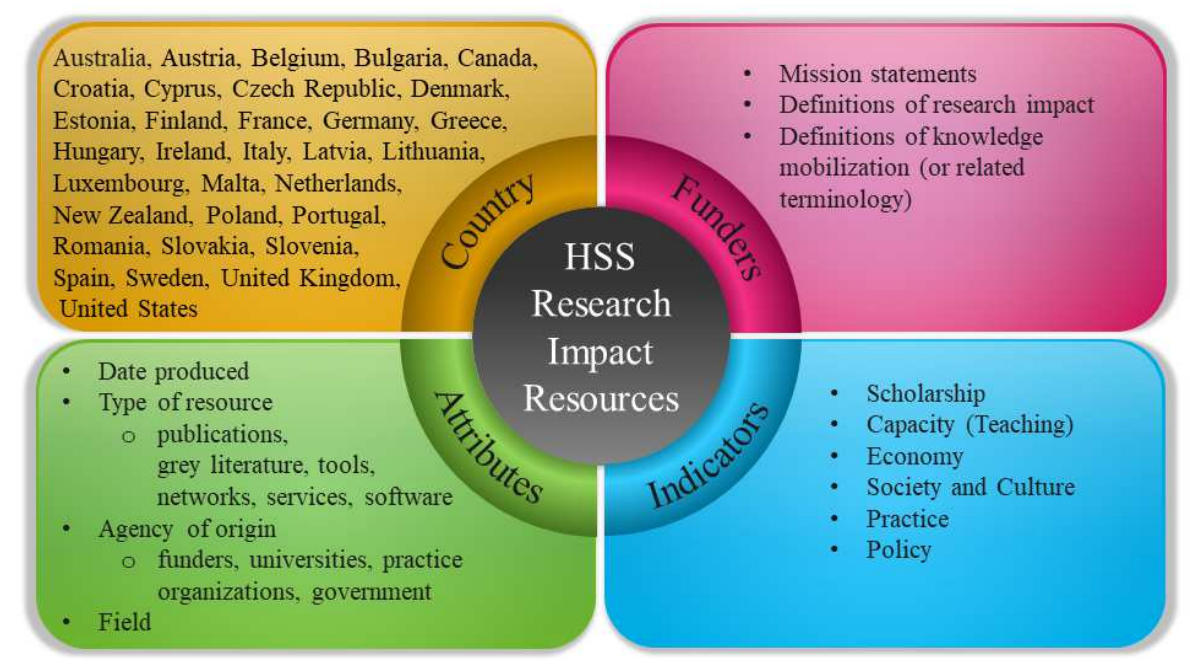

Fig. 1: Conceptual framework to classify and analyze types of research impact resources (adapted from Cooper, 2014) 
Tetroe et al. (2008) conducted a study of 32 health funding agencies and concluded, in relation to $\mathrm{KMb}$ and impact agendas, that "the largest looming barrier to advancing the KT agenda is the lack of conceptual clarity regarding what is meant by $\mathrm{KT}$ and what a commonly accepted framework might look like" (p. 152). We had a similar interest in determining the extent and clarity regarding social science funders' conceptualization of research impact and $\mathrm{KMb}$ or related terms and whether best practices and capacity-building resources might be available to aid researchers in meeting these new demands of research impact (which Watermeyer (2014) called the new academic capital). The third area of our conceptual framework explored the attributes of research impact resources uncovered in relation to date produced, type of resource, agency of origin (funders; governments; university, practice organizations, or international organizations such as the Organisation for Economic Cooperation and Development, and; and disciplinary field). The fourth category of our conceptual framework narrowed the broader results of the environmental scan to analyze HSS research impact indicators in relation to the framework developed by the Canadian Federation for the Humanities and Social Sciences (2014).

\section{Method}

Environmental scans are not simply ad hoc online searches; rather, Conway (2009) highlighted that environmental scanning is formal and systematic, using "formal methodologies for obtaining information for a specific purpose" (p. 2). The sample included 32 countries: Australia, Canada, New Zealand, United Kingdom, the United States and those countries which are members of the European Union (Austria, Belgium, Bulgaria, Croatia, Cyprus, Czech Republic, Denmark, Estonia, Finland, France, Germany, Greece, Hungary, Ireland, Italy, Latvia, Lithuania, Luxembourg, Malta, Netherlands, Poland, Portugal, Romania, Slovakia, Slovenia, Spain and Sweden).

Our scan used JSTOR to uncover peer reviewed journal articles and we utilized Google and Google Scholar to discover grey literature. We conducted the environmental scan using systematic search strings produced by combinations and permutations of key terms based on individual database thesauri (Table 1) in consultation with research librarians.

Table 1: Keywords used to produce systematic search strings

\begin{tabular}{llllll}
\hline Discipline & Organization type & Measure synonym & Research synonym & Impact synonym & Type of resource \\
\hline Humanities social & Research funder & Measure & Research & Impact & Framework \\
sciences & Government & Evaluate & Academic & Benefits & Assessment \\
& Intermediary & Assess & Evidence & Excellence & Indicators \\
& University & Capture & Best practice & & Standards \\
& & KT knowledge & & Metrics \\
& & Mobilization & & \\
\hline
\end{tabular}

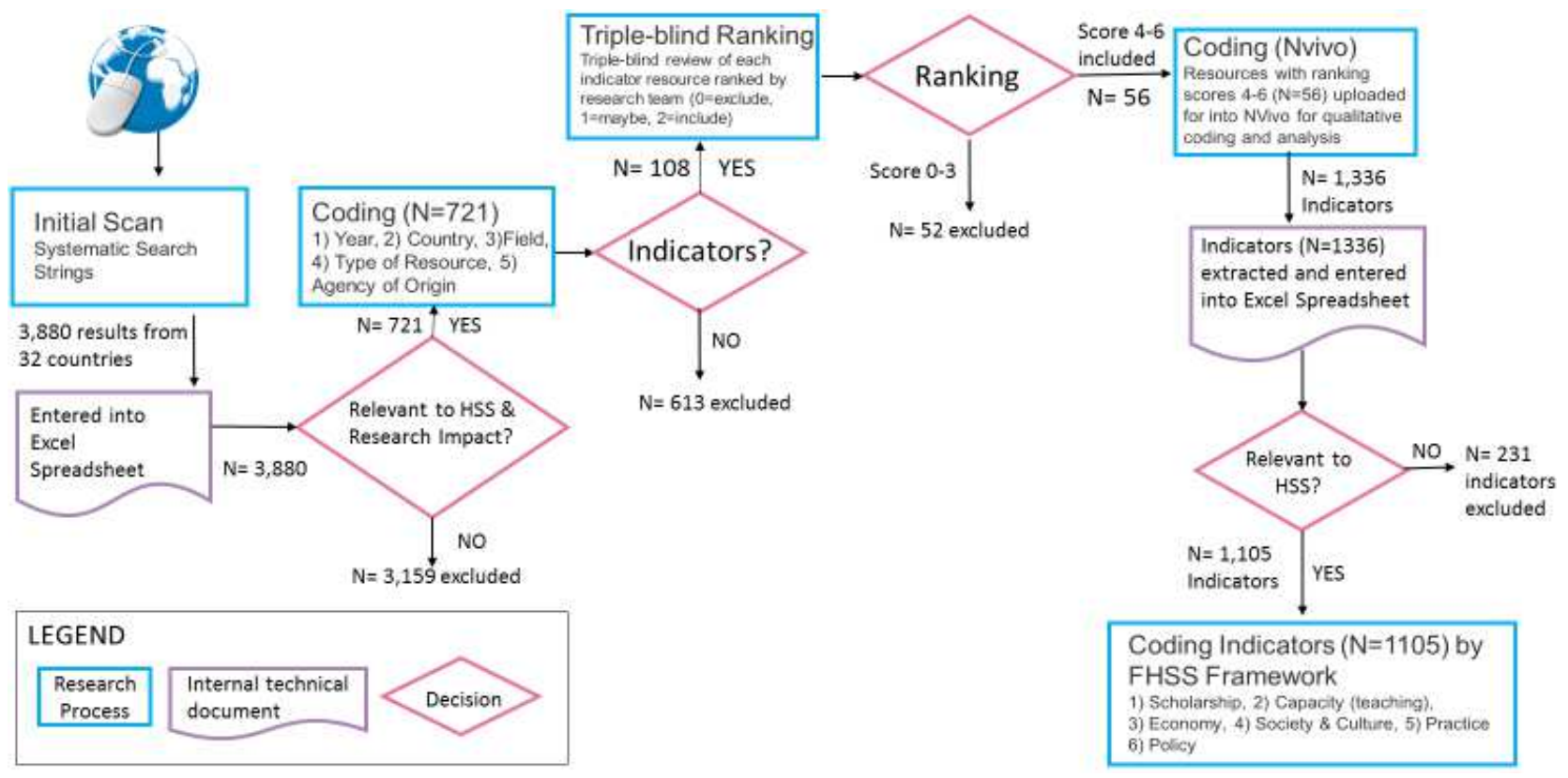

Fig. 2: Methodological approach to conducting environmental scan 
There were two phases of analysis. The first analyzed resources in relation to the conceptual framework. The second conducted in-depth analysis and coding for research impact indicators identified through the environmental scan. Figure 2 summarizes our methodological approach in relation to these two phases.

The initial search yielded 3,880 results based on our systematic search strings. We recorded specific Boolean searches with database, number of results and notes on the search and we analyzed each of the 3,880 results to determine inclusion or exclusion based on the conceptual framework and research questions. For instance, we excluded frameworks that were not relevant to HSS. We excluded 3,159 results, leaving a sample of 721 research impact resources to be included for coding and further analysis. These 721 results were coded in Excel in relation to the major areas of the conceptual framework including country, field, type of resource and agency and whether the resource contained discrete indicators to assess research impact. We calculated descriptive statistics to investigate trends.

One of our primary objectives was to amass HSS research impact indicators; consequently, we conducted further analysis of the 108 resources that had specific indicators using the Canadian Federation for the Humanities and Social Sciences (FHSS) Research Impact Framework: scholarship, capacity building (through teaching and mentoring), economy, society and culture and practice and policy. To determine which of these 108 resources should be included in an in-depth analysis, we conducted blind rankings by three members of our research team and allotted scores based on the resource's relevance to the study: $0=$ exclude, $1=$ maybe include, 2 $=$ include. We tallied scores and then excluded results with totals between 0 and 2 , included results with totals between 4 and 6 and further discussed results with ratings of 3 or 4 to decide whether to include them for indicator analysis. We uploaded each resource ranked highly relevant $(\mathrm{N}=56)$ into NVivo for further analysis and we coded indicators from each resource, which yielded 1,336 discrete indicators. Of these, we discarded 231 indicators for one of the following four reasons:

- The indicator was one word, which made extrapolation about the meaning difficult without the full article

- The indicator used acronyms that made understanding difficult

- The indicator was duplicated from the same document

- The indicator dealt with non-research-related activities

After excluding 231 indicators, we coded 1,105 indicators according to the FHSS framework (2014) in relation to scholarship, capacity, economy, society and culture, practice and policy.

\section{Limitations}

Findings from this study are based on data collected from peer-reviewed journals, grey literature and funder websites, therefore, it is possible that items were missed if they were not published online. Moreover, databases and search engines are optimized to retrieve pre-defined, specific and precise keywords. For those instances, one must know exactly what words to use and the search result for these words will be very precise and accurate. Therefore if one does not know what words to use in the search then traditional search tools will not help. We mitigated this risk by creating a list of keywords in consultation with our institution's research librarians. Finally, one of the purposes of these analyses was to identify the types of resources and indicators used and to create a detailed listing of different types of resources and indicators researchers could use to track their own work. A limitation of this part of the study was that these resources and indicators have not been vetted for quality (we have included and categorized all we found). In addition, different views on how the study grouped indicators and resources will undoubtedly arise; however, this scan represents a first stage to provide a baseline of resources and indicators that need to be further explored by peer-review panels of HSS researchers. However, this was still a necessary first step, with the next step of this work being the need to assemble multi-stakeholder panels in particular areas to conduct further work exploring the various groups of indicators in relation to the level of application that might be appropriate (individual, group, institutional, national), as well as creating instructions for researchers to consider when applying a particular indicator. This suggested next step, which was beyond the scope of this work, was the process followed to create an extensive typology of indicators in the health sector in Canada (Frank et al., 2009). Notwithstanding these limitations, this mapping exercise makes a valuable contribution to the field and serves to support academic stakeholders translate, discuss and adapt research in a variety of societal contexts.

\section{Findings}

We conducted analysis for descriptive statistics based on the research questions and conceptual framework.

\section{Social Science Research Funding Agencies Definitions and Conceptualizations of Research Impact}

To frame the discussion around research impact and $\mathrm{KMb}$ - and as a precursor to the environmental scan of 32 countries - our team visited websites of the HSS funding agencies for each country to see how funders were defining $\mathrm{KMb}$ and research impact. Our rationale was that funders influence the research infrastructure and focus in each country through requirements and expectations for researchers. Some countries had more than one research funding agency relating to HSS, so we visited a total of 39 funding agency websites. We explored two aspects of each 
funding agency: (a) its mission statements in relation to $\mathrm{KMb}$ and impact and (b) definitions of $\mathrm{KMb}$ and research impact to explore how research funders were conceptualizing and operationalizing expectations of these concepts for researchers.

Over $50 \%(\mathrm{~N}=22,56 \%)$ of the agencies included something relating to $\mathrm{KMb}$ and the potential benefits of research to the wider society in their mission statements. However, only three funding agencies had definitions of research impact ( $8 \%$ of funding agencies) and only five had some type of definition of $\mathrm{KMb}$ (13\% of funding agencies). Table 2 shows how social science funding agencies are defining $\mathrm{KMb}$ and research impact.

These definitions capture the potential for research to benefit broader society and move beyond academia; however, many are quite general. Canada's Social Sciences and Humanites Research Council has a comprehensive definition that emphasizes the reciprocal and complementary flow of knowledge between different stakeholder groups. Sweden also includes an interesting view that the primary role of research communication in relation to its potential contribution to enlightened and critically thinking citizens that characterize democratic societies. Moreover, the Swedish Research Council also highlights the important role that research should play in promoting dialogue.

\section{Research Impact Resources and Their Attributes}

The environmental scan for research impact resources for HSS across 32 countries identified 721 research impact resources. We were interested in mapping the different kinds of research impact resources that have been produced. The types uncovered by the environmental scan included journal articles, grey literature, government publications, books and book chapters, services, networks, conference-related resources, project-based resources, magazines, repositories and software in order to trace influence (for instance, to assess social media platforms such as Twitter and Facebook). Peer-reviewed journal articles made up $45 \%$ of the resources identified and included a range of impact frameworks, studies and indicators. The second most prominent category included grey literature (35\%), followed by governmental publications $(6 \%)$.

Table 2: Funders' definitions of $\mathrm{KMb}$ and research impact Definitions of $\mathrm{KMb}$ and research impact from HSS funders

Australian Research Council

Social Sciences and Humanities Research Council, Canada

Deutsche Forschungsgemeinschaft, Germany

Netherlands Organization for Scientific Research (NWO)

Vetenskapsrādet, Sweden

Economic and Social Research Council, UK

Arts and Humanities Research Council, UK
Knowledge transfer: Knowledge transfer is deliberately embedding

knowledge for use in a context beyond the researcher's own sphere (2015).

Research impact: is the demonstrable contribution that research makes to the economy, society, culture, national security, public policy or services, health, the environment, or quality of life, beyond contributions to academia (2015).

Knowledge mobilization: The reciprocal and complementary flow and uptake of research knowledge between researchers, knowledge brokers and knowledge users - both within and beyond academia - in such a way that may benefit users and create positive impacts within Canada and/or internationally, and, ultimately, has the potential to enhance the profile, reach and impact of Social Sciences and Humanities Research (2016).

Knowledge transfer: The term 'knowledge transfer' refers to a transfer of knowledge between research and industry or the general public (2016).

Knowledge utilisation: NWO believes that scientific knowledge and skills should be available and used outside of academia and/or in other scientific disciplines, in other words that knowledge utilisation should take place (n.d.).

Research communication: Research communication is needed so that knowledge generated by research can be of benefit to society (2013).

Objectives of research communication: The expansion of democracy created in a society of enlightened and critically thinking citizens is the overarching object of research communicating. Other goals are to illustrate and increase understanding of the importance of research to society. It is also important to promote dialogue and communication within the research community and to ensure that the results of research reach the areas in society where they can be useful, for example within education, healthcare and in trade and industry (2013).

Research impact: The demonstrable contribution that excellent research makes to society and the economy. Research impact embraces all the diverse ways that research-related skills benefit individuals, organisations and nations. These include:

- Fostering global economic performance, and specifically the economic competitiveness of the United Kingdom

- Increasing the effectiveness of public services and policy

- $\quad$ Enhancing quality of life, health and creative output (n.d.). Research impact: By impact we mean the "influence" of research or its "effect on" an individual, a community, the development of policy, or the creation of a new product or service. It relates to the effects of research on our economic, social and cultural lives (n.d.). 


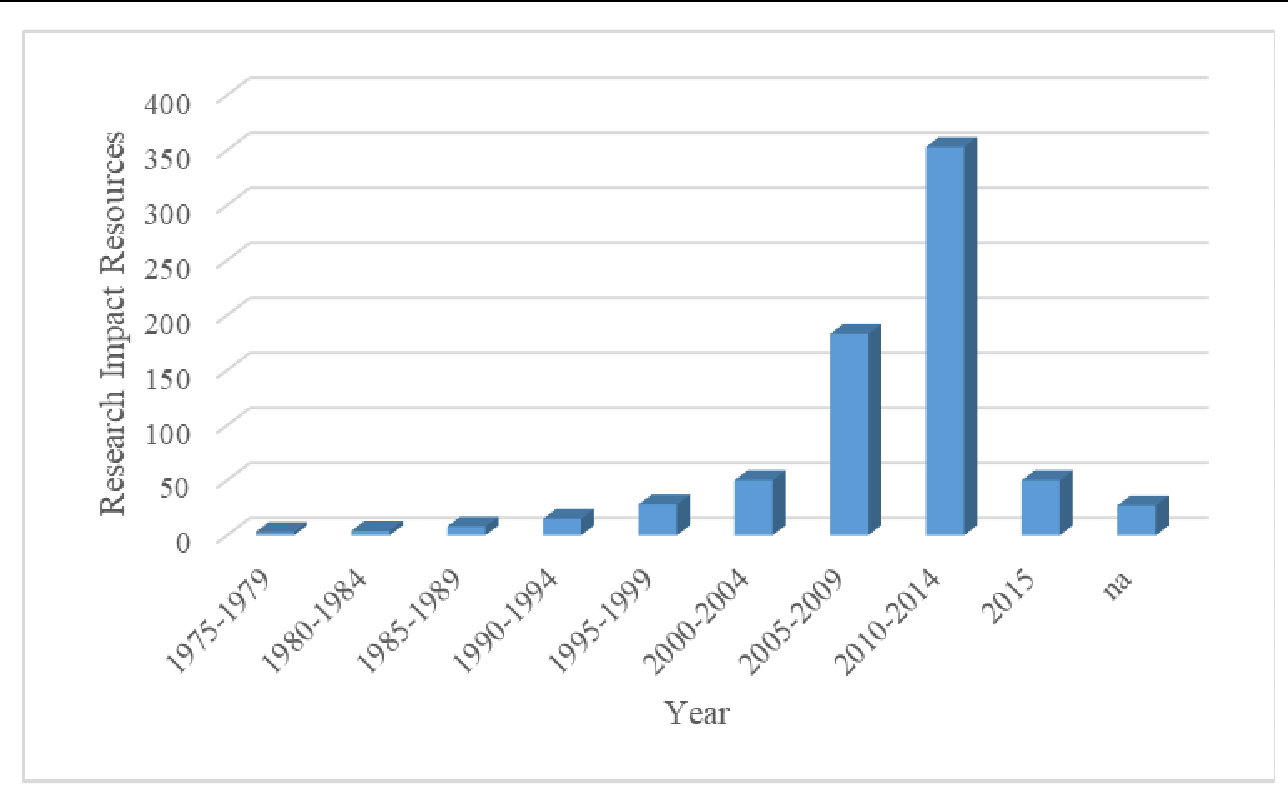

Fig. 3: Date of origin of research impact resources $(N=721)$

Very few tools were tailored to help researchers meet the rising demands from funders and governments (less than $9 \%$ of the total sample). Research impact resources were not always free; some (such as journal articles and software) required licenses or subscriptions. However, some toolkits and resources were freely available to assist researchers in their efforts to mobilize research and trace the subsequent influence of their work.

There has been a veritable explosion of research impact resources in the past decade (Fig. 3). Eightyone percent of the resources have arisen since 2005, with over half the research impact resources originating in the past 5 years.

This trend, in and of itself, shows the growing prominence of research impact agendas across the globe.

We were also interested in exploring what kinds of agencies were producing research impact resources. Universities $(43 \%)$ have created the most research impact resources, followed by practice organizations $(16 \%)$, government organizations $(13 \%)$, funders $(9 \%)$, international organizations such as the OECD (4\%) and other types of agencies (16\%). Practice organizations occupy a translational role between researchers and community members, so more work might include a deeper investigation of how those organizations and their efforts have increased research impact in various communities. We also coded results in relation to field. Our search terms privileged HSS; however, if resources seemed applicable across disciplines, we also included these results. Education represented approximately 5\% of resources and business, economics and arts also accounted for a small percentage of resources (less than $2 \%$ each).

In our analysis, the United Kingdom $(\mathrm{N}=139)$, Canada $(\mathrm{N}=109)$, the United States $(\mathrm{N}=76)$ and the
Netherlands $(\mathrm{N}=50)$ had the most research impact resources. Due to the move to the REF in the United Kingdom, it is perhaps not surprising that they had the largest proportion of research impact resources. Countries including Australia $(\mathrm{N}=34)$, Spain $(\mathrm{N}=32)$, Italy $(\mathrm{N}=30)$, Germany $(\mathrm{N}=25)$, Denmark $(\mathrm{N}=22)$ and Austria $(\mathrm{N}=18)$ also produced research impact resources. International organizations (which include multiple partner countries such as the OECD) also featured prominently in the distribution of research impact resources $(\mathrm{N}=105)$.

\section{Further Analysis of Indicators}

Indicators used to measure research impact were an important focus of the environmental scan. Of the 721 research results, $108(15 \%)$ included specific indicators or metrics. We extracted 1,105 indicators from these results. We then coded all 1,105 indicators according to the Canadian Federation for the Humanities and Social Sciences (FHSS) framework (Fig. 4): capacity building (through teaching and learning), economy, practice, policy, scholarship, and society. Our analysis expanded the FHSS conceptual framework in two ways: (a) by separating practice and policy categories and (b) by creating further disaggregated groups under each of the major categories. Figure 5 shows the relative proportion of the indicators in relation to the FHSS categories of research impact.

The largest proportion of discrete indicators was in relation to scholarship (38\%), followed by capacity for teaching (23\%), economy (14\%), society and culture $(9 \%)$, practice $(13 \%)$ and policy $(3 \%)$. It is perhaps unsurprising that so few indicators related to practice and policy because the literature has lamented these areas as the most difficult to assess. 


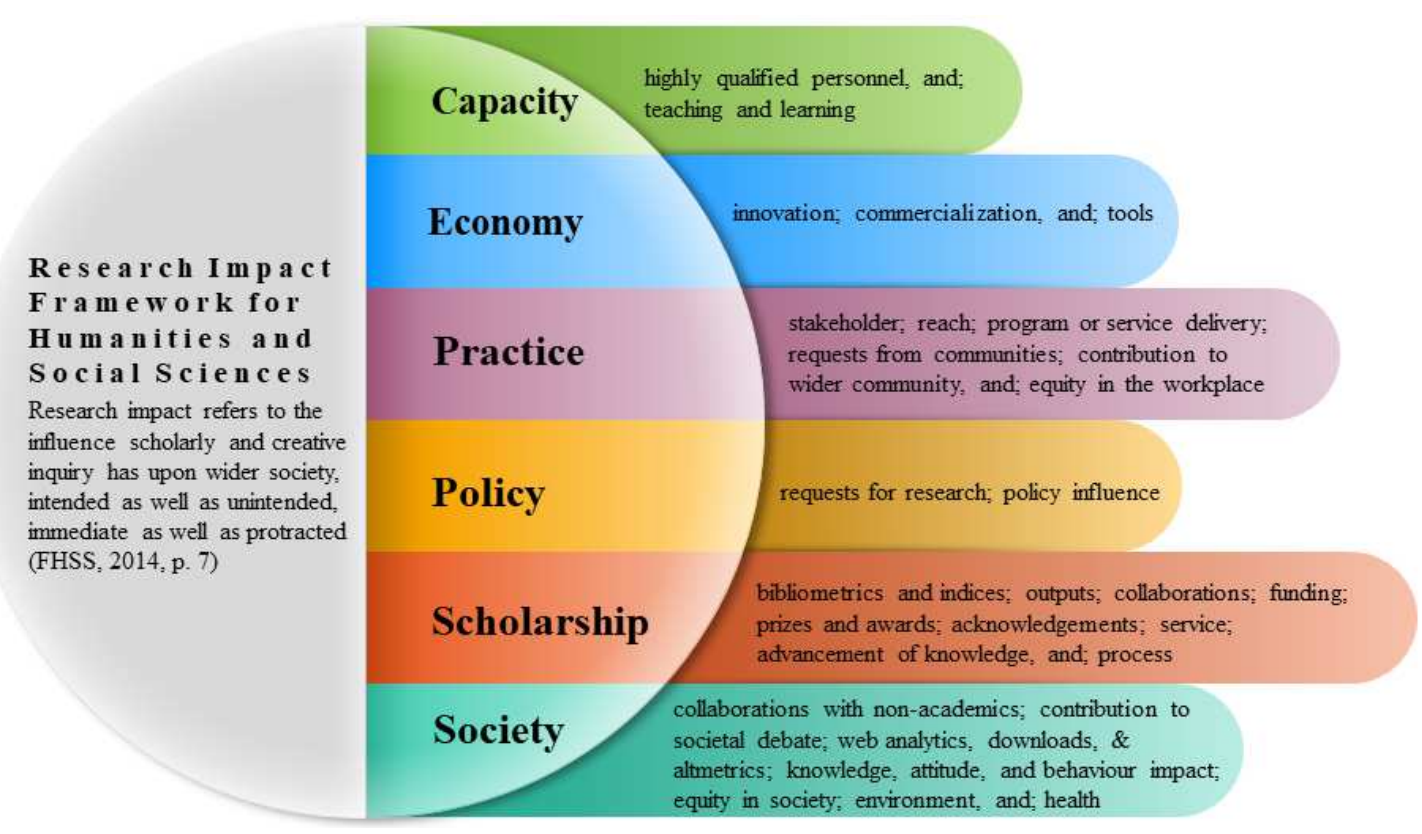

Fig. 4: Research impact framework for humanities and social sciences (adapted from FHSS, 2014)

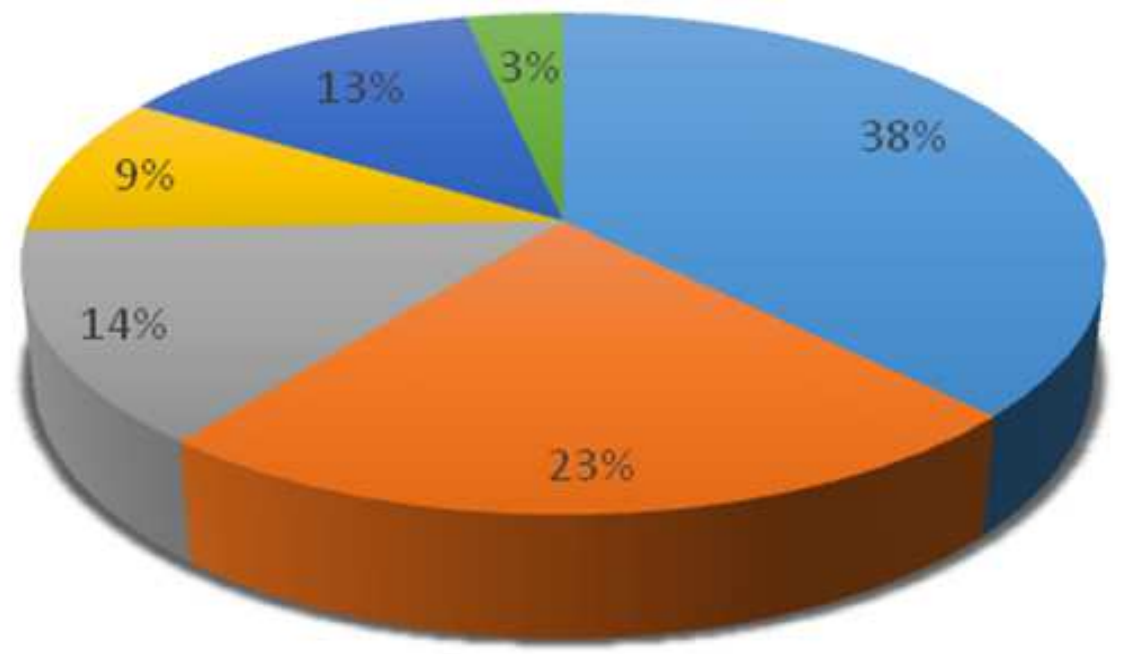

Scholarship

Capacity

(Teaching)

Economy

Society and culture

Practice

Policy

Fig. 5: Proportion of indicators based on FHSS impact framework categories

We were also interested in the relative proportion of indicators that measured quantity versus quality. Therefore, we also coded all 1,105 indicators in relation to whether they measured the quantity or quality of something. Seventy-eight percent $(\mathrm{N}=858)$ of the indicators related to quantity and $22 \%(\mathrm{~N}=247)$ related to quality.

\section{Discussion}

A number of issues are emerging from research impact agendas occurring globally. We organize these issues in relation to the research questions.
Many Social Science Research Funding Agencies Lack Clear Definitions and Conceptualizations of Research Impact and $\mathrm{KMb}$

Unintended consequences of narrow conceptualizations of research impact are being uncovered globally, as some studies reveal "that an emphasis on demonstrating impact may actually hijack or subjugate the process of achieving impact and potentially even dilute or inhibit the positive effects of research" (Watermeyer, 2014, p. 362). Therefore, funders having unclear definitions and guidelines surrounding research impact and expectations for 
translation efforts is problematic because researchers need to better understand how funders operationalize these concepts so they can align their approaches to $\mathrm{KMb}$ and impact in their applications and projects. In a related study (Cooper et al., 2015) we uncovered how HSS funders in OECD and BRICs countries are operationalizing $\mathrm{KMb}$ and research impact. We conducted an environmental scan of 45 HSS funding agency websites and extracted data using a coding manual and common data collection spreadsheet to assess the existence of 60 elements organized in relation to how agencies are promoting (through requirements for researchers) and supporting (through agency initiatives) $\mathrm{KMb}$ and research impact. Over half $(53 \%)$ of the agencies we analyzed received failing scores in relation to their $\mathrm{KMb}$ and research impact efforts. The Economic and Social Research Council (UK), Social Sciences and Humanites Research Council (Canada), and Netherlands Organization for Scientific Research were the top three scoring (95\%; 83\%; 83\%) agencies respectively. Agencies with unclear definitions or conceptualizations of $\mathrm{KMb}$ and research impact should look to the above funders who are front runners in the field.

\section{Numerous Indicators Exist to Measure Research Impact, however, Caution should be used when Determining Appropriate Measures}

The enduring uncertainty surrounding research impact agendas is founded in the recognition that the field of research impact and many of the metrics and indicators proposed across disciplines are underdeveloped. This is especially problematic in jurisdictions where performance-based research funding systems have tied hard dollars to impact assessments. However, regardless of the widespread consensus that indicators and metrics do not adequately capture impact (especially when comparing across diverse disciplines), funding agencies and national bodies in many countries are marching forward (in the United Kingdom and Australia, to name two). Because of these developments, it is imperative that HSS researchers across the globe engage in the debate to influence its operationalization. In addition, an over-reliance on research impact has begun to change researchers' behaviours in the United Kingdom in undesirable ways. The Metric Tide (Wilsdon et al., 2015) is a comprehensive independent review of the role of metrics in research assessment and management conducted by a multidisciplinary group of experts in the United Kingdom. The authors highlighted five issues arising from indicator effects. The first is strategic behavior and goal displacement. Studies have indicated that funding and evaluation such as the REF are leading to goal displacement in many countries, where the goal becomes scoring highly on the assessment exercise. The second issue, effects on interdisciplinary, also has negative effects on goal displacements. Task reduction is also problematic in that certain types of activities that are not evaluated in performance-based funding systems (such as teaching and outreach) are abandoned to focus on publishing in particular journals that will increase standing in the performance assessment. The fourth effect of indicator uses is in relation to effects on institutions, where some evidence shows alignment with hiring practices or promotion between institutions and funding criteria. The last is effects on knowledge production that "point to a discrepancy between the importance of indicators in evaluation practices according to academics and their own judgement of the accuracy of certain measures" (Wouters et al., 2015, p. vii). Although Wilsdon et al. (2015) recognized the positive potential of systems that encourage collaboration that might increase the impact of research in communities, they also cautioned field and national funders about the application of such metrics across different institutions and disciplines.

The Wilsdon et al. (2015) report ultimately advocated for what the authors referred to as "responsible metrics as a way of framing appropriate uses of quantitative indicators in the governance, management and assessment of research" (x). They identified five dimensions through which to understand responsible metrics:

- Robustness: Basing metrics on the best possible data in terms of accuracy and scope

- Humility: Recognising that quantitative evaluation should support-but not supplant-qualitative, expert assessment

- Transparency: Keeping data collection and analytical processes open and transparent so those being evaluated can test and verify the results

- Diversity: Accounting for variation by field and using a range of indicators to reflect and support a plurality of research and researcher career paths across the system

- Reflexivity: Recognising and anticipating the systemic and potential effects of indicators and updating them in response. (p. x)

Despite concerns with the use of metrics and accountability measures tied to research impact assessments, the underlying purpose of research impact efforts (if one takes them at face value) is positive. Research impact is about building collaborative partnerships to solve challenging societal problems and to inform debates in democratic societies. Impact, in the end, is about the people in communities with whom researchers can collaborate on shared goals, not academic publications in high-impact journals that are not read outside disciplinary silos within the academy. In addition to positive and negative consequences of research impact agendas (including both intended and unintended consequences), much is still unknown because these agendas, from a historical perspective, are still in their infancy. 
A Guidebook and Taxonomy of Research Impact Resources and Indicators were created to Help Researchers

Out of the 721 research impact resources, 87 resources were specifically designed to support researchers in their knowledge mobilization and impact endeavors. We organized these resources by type and topic and included them in a supplementary guidebook. Major categories of resources included tools $(\mathrm{N}=56)$, organized by four subgroups (policy and politics, software and services, grant writing and research knowledge and mobilization); networks $(\mathrm{N}=17)$, including communities of practice and research networks; projects $(\mathrm{N}=10)$, focusing specifically on research impact, indicators and metrics; and open access repositories $(\mathrm{N}=4)$ that provide examples of research impact case studies and outputs. Due to the scope of this article, we have not provided highlights of these resources here.

A taxonomy of research impact indicators for humanities and social sciences was also developed as a result of the environmental scan. Due to similar types of indicators being listed, we collapsed the overall list of 1,105 indicators found from the literature into a list of 420 discrete indicators with multiple citations and sources and included it in the supplementary file for researchers to consider when contemplating indicators they might use for their grants or to assess $\mathrm{KMb}$ and impact on their projects.

\section{Conclusion}

This article heeds the call of Watermeyer (2014) by producing a cartography of impact indicators and resources for HSS researchers. Although exploratory, the article makes a necessary contribution to showing the ways in which impact is being conceptualized, operationalized and measured. It also demonstrates the lack of available substantive resources for researchers and speaks to the need for capacity building if researchers are to be successful working with nonacademic audiences and increasing the mobilization and impact of their work. What is clear from the veritable explosion of research impact materials in the past decade and the increasing number of performance-based research funding systems arising globally is that research impact will be a defining factor of research infrastructure, funding and landscapes across the world for the foreseeable future. Although we are optimistic about the value of encouraging researchers to work with non-academic audiences to tackle complex societal issues, we caution funders and universities not to use large-scale impact cases to determine funding because, at present, research impact indicators are not satisfactorily developed to make funding decisions. However, we do believe that many benefits can arise from a continued focus on developing capacity for knowledge mobilization, especially where diverse stakeholders collaborate to tackle complex societal issues.

\section{Acknowledgement}

This research was supported by the Canadian Federation for the Humanities and Social Sciences.

\section{Author's Contributions}

Samantha Shewchuk: Samantha Shewchuk completed data collection and analyses, helped write the manuscript, and created the supplementary files.

Amanda Cooper: Dr. Cooper conceptualized the study and helped write the manuscript.

\section{Ethics}

Samantha Shewchuk and Dr. Amanda Cooper declare they have no conflicts of interest.

\section{References}

Arts and Humanities Research Council, n.d. Impact summary and pathways to impact frequently asked questions.

Australian Research Council, 2015. Glossary of terms for research impact. Australian Research Council.

Chandler, C., 2014. What is the Meaning of Impact in Relation to Research and Why Does it Matter? A View from Inside Academia. In: Achieving Impact in Research, Denicolo, P. (Ed.), Thousand Oaks, CA: Sage, pp: 1-15.

Conway, M., 2009. Environmental Scanning: What it is and How to Do it. 1st Edn., Hotham Hill, Australia.

Cooper, A., S. Shewchuk and S. MacGregor, 2015. Social science funding agencies' promotion and support of knowledge mobilization and research impact: an international study. Kingston: RIPPLE research report. www.ripplenetwork.ca

Cooper, A., 2014. Knowledge mobilisation in education across Canada: A cross-case analysis of 44 research brokering organisations. Evidence \& Policy: A J. Research, Debate Practice, 10:29-59. DOI: $10.1332 / 174426413$ X662806

Cooper, A., B. Levin and C. Campbell, 2009. The growing (but still limited) importance of evidence in education policy and practice. J. Educational Change, 10: 59-171. DOI: $10.1007 / \mathrm{s} 10833-009-9107-0$

Davies, H., 2003. Policy Development: Making Research Count in Child Wefare. In: Child Welfare: Connecting Research, Policy and Practice, Kufeld, K. and B. McKenzie (Eds.), Walter, Ontario: Canada.

Deutsche Forschungsgemeinschaft, 2016. Knowledge transfer. 
Economic and Social Research Council, n.d. What is impact?

Federation for the Humanities and Social Sciences, 2014. The impacts of humanities and social science research. Federation Humanities Soc. Sci., Canada.

Frank, C., R. Battista, L. Butler, M. Buxton and N. Chappel et al., 2009. Making an impact: A preferred framework and indicators to measure returns on investment in health research-Summary of the report.

Netherlands Organization for Scientific Research, n.d. Knowledge utilisation and PPP.

Nutley, S.M., I. Walter and H.T.O. Davies, 2007. Using EVIDENCE: How research can Inform Public Services. 1st Edn., Policy Press, Bristol, ISBN-10: 1861346646, pp: 363.

Social Sciences and Humanites Research Council, 2016. Definition of terms.

Tetroe, J., I. Graham, R. Foy, N. Robinson and M. Eccles et al., 2008. Health research funding agencies support and promotion of knowledge translation: An international study. Milbank Quarterly, 86: 125-155.
Vetenskapsrādet, 2013. Research communication.

Watermeyer, R., 2014. Issues in the articulation of "impact": The responses of UK academics to "impact" as a new measure of research assessment. Stud. Higher Educ., 39: 359-377.

DOI: $10.1080 / 03075079.2012 .709490$

Wilsdon, J., L. Allen, E. Belfiore, P. Campbell and S. Curry et al., 2015. The metric tide: Report of the independent review of the role of metrics in research assessment and management. Bristol: Higher Education Funding Council for England.

Wouters, P., M. Thelwall, K. Kousha, L. Waltman and S. De Rijcke et al., 2015. The metric tide: Literature review. Proceedings of the Supplementary Report I to the Independent Review of the Role of Metrics in Research Assessment and Management, (RAM' 15), London, UK. 